\title{
Controllable quantum spin precession by Aharonov-Casher phase in a conducting ring
}

\author{
Shun-Qing Shen ${ }^{\mathrm{a})}$ \\ Department of Physics, The University of Hong Kong, Pukfulam Road, Hong Kong, China \\ Zhi-Jian Li \\ Institute of Physics, Chinese Academy of Sciences, Beijing 100080, China \\ Zhongshui Ma \\ State Key Laboratory for Mesoscopic Physics and Department of Physics, Peking University, \\ Beijing 100871, China
}

(Received 26 June 2003; accepted 3 December 2003)

\begin{abstract}
We investigate quantum spin transport in a structure of a conducting ring embedded in a textured electric field with two leads, and obtain an exact solution for the problem. The spin precession induced by the Aharonov-Casher phase is studied. It is shown that the spin-polarized current and its polarization orientation can be controlled by the electric field. As a result the modulated polarization orientation is a function of the geometric phase which originates from spin-orbital interaction in the ring. (c) 2004 American Institute of Physics. [DOI: 10.1063/1.1644914]
\end{abstract}

In recent years quantum spin transport has attracted considerable interest because of its potential application in semiconductor electronics and quantum computation. ${ }^{1-3}$ How to control or modulate the spin-polarized current is an important step in the investigation of the spin coherence in electronic systems. Datta and Das ${ }^{4}$ proposed a spin field-effect transistor (FET), in which the spin polarization of charge carriers precesses when the charge carriers are transmitted through a two-dimensional (2D) semiconductor channel between a ferromagnetic spin injector and a ferromagnetic spin collector. It is well known that, in semiconductor heterostructures, spin-orbit or Rashba interaction creates spinorbit splitting of the conduction electron energy band. Such splitting produces spin precession while the electrons pass through the semiconductor channel. Experimentally, Rashba coupling can be adjusted by an electric field such that a spinpolarized current injected from the source can be spin dependently modulated on its way to the drain by an external gate. ${ }^{5,6}$ Other proposals were reported recently. ${ }^{7-11}$ However, more sophisticated structures are conceivable in which current modulation could arise from spin interference.

The conventional Aharonov-Bohm (AB) effect is anticipated to modulate the charge current. A nonuniform magnetic field may also control the polarization orientation of the spin current. ${ }^{12}$ The Aharonov-Casher (AC) effect ${ }^{13}$ originates from spin-orbit coupling between the moving magnetic polar and electric fields. It is expected to implement the spin current modulation manifested by the AC effect. Quantum coherent transport in a structure of an $\mathrm{AC}$ ring with two leads has been discussed by many authors. ${ }^{14-18}$ It was found that $\mathrm{AC}$ flux can lead to interference phenomena such as $\mathrm{AB}$ flux with observable AC oscillation in the conductance. With the electric field as a parameter, it is expected that one can study the precession of spin current for electron interference manifested by the geometric properties of the ring. In this letter we establish a connection between the quantum spin polarization orientation and spin precession in quantum

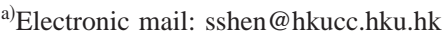

transport in an $\mathrm{AC}$ ring. It is shown that spin precession can be controlled through the external electric field by means of the AC effect. We present an exact solution for the problem and focus on the spin aspect of quantum transport. Assume the ring is embedded in a symmetrically textured electric field with arbitrary tilt angle as shown in Fig. 1. In the AC ring, the magnetic moment of charge carriers is influenced by the electric field through spin-orbit interaction. There is energy splitting between spin-up and -down electrons. The local spin orientations on the ring are determined by spin cyclic evolution over the ring. When electrons travel through each arm of the ring, the wave functions of electrons acquire a spin-dependent Aharonov-Anandan (AA) phase, ${ }^{19}$ which is the geometric part of the $\mathrm{AC}$ phase. Without specifying the direction of spin-polarized injection, we calculate the spindependent transmission coefficients analytically. Within the Landauer framework of ballistic transport, ${ }^{20}$ it is shown that the spin-polarized current and its modulation of polarization can be controlled by the electric field via the AC phase. Our result shows that the $\mathrm{AC}$ ring can act as a tunable spin switch by adjusting the electric field.

In the ring, the one-particle Hamiltonian for noninteracting electrons with momentum $\mathbf{p}$ is given by

$$
H=\frac{1}{2 m}\left(\mathbf{p}-\frac{\mu}{2 c} \boldsymbol{\sigma} \times \mathbf{E}\right)^{2},
$$

where $\mu=g \mu_{B}$ is a magnetic moment of the charge carrier,

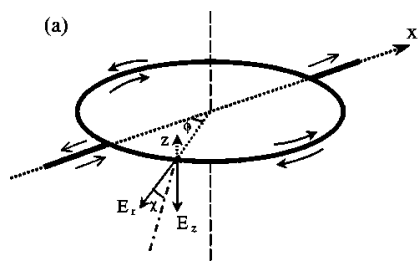

(b)

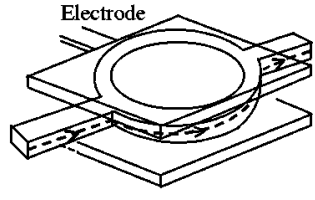

FIG. 1. (a) Effect of a symmetrically textured electric field on the AC ring. The arrows indicate the incident, transmitted, and reflected current flow. (b) Spin transistor geometry: an AC ring with two leads connected to the ferromagnetic source and drain sandwiched between two electrodes. 
$\mu_{B}$ is the Bohr magneton, $g$ is the gyromagnetic factor, $c$ is the velocity of light in vacuum, and $\sigma_{i}(i=1,2,3)$ is the Pauli matrix. The linear term of the electric field in the Hamiltonian, $\boldsymbol{\sigma} \cdot(\mathbf{E} \times \mathbf{p})$, represents the spin-orbit coupling. In the cylindrical coordinate, the textured electric field can be written as $\mathbf{E}=E(\cos \chi \hat{\mathbf{r}}-\sin \chi \hat{\mathbf{z}})$. The eigenfunctions of Hamiltonian (1) are given by $\Psi_{n, \pm}=1 / \sqrt{2 \pi} e^{\text {in } \phi} \xi_{ \pm}$with

$\xi_{+} \equiv\left(\cos \frac{\beta}{2}, e^{i \phi} \sin \frac{\beta}{2}\right)^{T}, \quad \xi_{-} \equiv\left(\sin \frac{\beta}{2},-e^{i \phi} \cos \frac{\beta}{2}\right)^{T}$,

where $T$ stands for the transpose of the matrix, and \pm denotes spin up and down along the direction of unit vector $\Omega=(\sin \beta \cos \phi, \sin \beta \sin \phi, \cos \beta) . \beta$ is defined by the function $\tan \beta \equiv \omega_{1} /\left(1+\omega_{3}\right)$ with $\omega_{1} \equiv(\mu E a / \hbar c) \sin \chi$ and $\omega_{3}$ $\equiv(\mu E a / \hbar c) \cos \chi$. The corresponding eigenvalues are $E_{n, \pm}=\hbar^{2} / 2 m a^{2}\left(n-\Phi_{\mathrm{AC}}^{ \pm} / 2 \pi\right)^{2}$, where $n$ is an integer. $\Phi_{\mathrm{AC}}^{ \pm}=-\pi \pm \phi_{0}\left[\phi_{0}=\pi \sqrt{\omega_{1}^{2}+\left(1+\omega_{3}\right)^{2}}\right]$ are the AC phases which are acquired while the two spin states $\Psi_{n, \pm}$ evolve in the ring in the presence of the electric field. ${ }^{13}$

Assume that electrons are free in two leads and have momentum $\hbar k$. The energy is given by $E=\hbar^{2} k^{2} / 2 m$. When an electron is transported along one arm in the clockwise direction from input intersection $\mathrm{A}$, it acquires $\mathrm{AC}$ phase $\Phi_{\mathrm{AC}}^{ \pm} / 2$ at output intersection B whereas the electron acquires phase of $-\Phi_{\mathrm{AC}}^{ \pm} / 2$ in the counterclockwise direction along the other arm. The total phase, therefore, is $\Phi_{\mathrm{AC}}^{ \pm}$, when the electron goes through the loop. In the ring the electric field may change the momenta of electrons into the same energy as that in the leads for two different spin states $\xi_{ \pm}$, i.e., $k_{1}^{ \pm}=k+\Phi_{\mathrm{AC}}^{ \pm} / 2 \pi a$ and $k_{2}^{ \pm}=k-\Phi_{\mathrm{AC}}^{ \pm} / 2 \pi a$, where subscripts 1 and 2 denote the clockwise and counterclockwise directions, respectively. $a$ is the radius of the ring. It is worth pointing out that $\left(k_{1}^{ \pm}-k_{2}^{ \pm}\right) \pi a=\Phi_{\mathrm{AC}}^{ \pm}$are independent of the momentum of incident electrons. The differences in phase lead two branches of wave functions to intervene at the output intersection ( $x$ ranges from 0 to $\pi a$ in two arms of the ring). The wave functions in two arms of the ring can be written as

$$
\begin{aligned}
& \Psi_{1}=\sum_{\alpha= \pm}\left(c_{1, \alpha} e^{i k x}+d_{1, \alpha} e^{-i k x}\right) e^{i \Phi_{\mathrm{AC}}^{\alpha} / 2 \pi a} \xi_{\alpha}\left(+\frac{x}{a}\right), \\
& \Psi_{2}=\sum_{\alpha= \pm}\left(c_{2, \alpha} e^{i k x}+d_{2, \alpha} e^{-i k x}\right) e^{-i \Phi_{\mathrm{AC}}^{\alpha} x / 2 \pi a} \xi_{\alpha}\left(-\frac{x}{a}\right) .
\end{aligned}
$$

We have assumed that a polarized electron is injected into the left electrode from the ferromagnetic source and travels in the $x$ direction. The corresponding spin state of the injected electron is $\Psi_{i}=(\cos \alpha, \sin \alpha)^{T} e^{i k x}$. Generally the wave functions in the two arms can be expressed in terms of the reflection and transmission of electrons. The wave functions of the electron in the left and right electrodes are

$$
\Psi_{l}=\Psi_{i}+\left(r_{\uparrow}, r_{\downarrow}\right)^{T} e^{-i k x}, \quad \Psi_{r}=\left(t_{\uparrow}, t_{\downarrow}\right)^{T} e^{i k x},
$$

where $r_{\sigma}$ and $t_{\sigma}$ are the spin-dependent reflection and transmission coefficients, respectively.

In the Landauer framework, ${ }^{20}$ the quantum mechanical transmission amplitude is related to two-probe conductance. The spin-dependent conductance through the ring may be expressed in terms of the transmission probability, $T_{\alpha}$

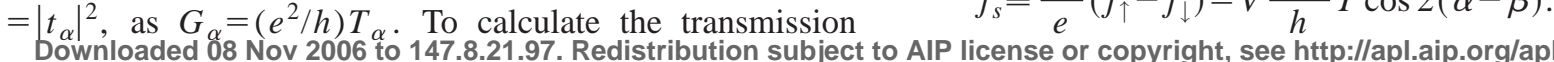

probability through the $\mathrm{AC}$ ring, we use the local coordinate system for each circuit such that the $x$ coordinate is taken along the electron current. ${ }^{17,21}$ The origin of each local coordinate is taken at each intersection. The choice of the origin of the coordinate is trivial because it only affects an extra phase factor of the transmission amplitude. Thus the Griffith boundary condition ${ }^{22}$ states that the wave function is continuous and that the current density is conserved at each intersection. After some tedious algebra, we obtain the transmission coefficient $t$,

$t=\frac{i \sin k \pi a \sin \phi / 2}{\sin ^{2} \phi / 2-(\cos k \pi a-i / 2 \sin k \pi a)^{2}}\left(\begin{array}{c}\cos (\alpha-\beta) \\ \sin (\alpha-\beta)\end{array}\right)$.

The corresponding transmission coefficient is given by

$T=\left|t_{\uparrow}\right|^{2}+\left|t_{\downarrow}\right|^{2}=\left|\frac{\sin k \pi a \sin \phi / 2}{\sin ^{2} \phi / 2-(\cos k \pi a-i / 2 \sin k \pi a)^{2}}\right|^{2}$.

The transmission probability was obtained for two special cases by Choi et al. ${ }^{17}$ For instance, when $\alpha=\beta / 2, c_{n,-}$ $=d_{n,-}=0$. In this sense only the component of $\xi_{+}$remains and there is no spin flip. Our general expression shows that the transmission probability is independent of the incident spin state. Both expressions in Eqs. (6) and (7) are determined by the AC phase, the spin and kinetic states of incident electrons, and the electric field. It should be noted that there is a special case where the transmission coefficients become zero which implies that all incident waves reflect from the ring when $\sin k \pi a=0$. To illustrate the main physics in the present problem, we present some numerical calculations for several physical quantities. For an InAs ring with $a=1 \mu \mathrm{m}$, the Fermi velocity $v_{F}$ is approximately 3 $\times 10^{7} \mathrm{~cm} \mathrm{~s}^{-1}$ and $k a \approx 60 .{ }^{16}$ It is interesting that the transmission probability is only determined by the decimal part of $k a$. The number is tunable for $k$ and the size of ring $a$. Thus we take $k a=60.239$ in the numerical calculations.

Employing the Landauer formula, the spin-resolved current is given by $j_{\sigma}=V G_{\sigma}=V\left(e^{2} / h\right) T_{\sigma}$. An interesting observation is

$$
j_{e} \equiv j_{\uparrow}+j_{\downarrow}=V \frac{e^{2}}{h} T,
$$

which is determined by the $\mathrm{AC}$ phase as well as by the radius of the ring. It depends on the energy of incident particles but is independent of the initial spin state. Therefore, the electric field can modulate the transmission charge current through the AC phase. In Fig. 2, we plot $j_{e}$ versus the magnitude $E$ and the direction angle $\chi$ of the electric field. For a specific size $a$ and incident momentum $k$, we find that the transmission charge current oscillates with the electric field for an arbitrary $\chi$. From two explicit expressions for $t_{\uparrow}$ and $t_{\downarrow}$ we observe that, after going through the AC ring, the electron evolves from the spin state $(\cos \alpha, \sin \alpha)$ into a new one $[\cos (\alpha-\beta), \sin (\alpha-\beta)]$. The spin current along the $S_{z}$ axis is given by

$$
j_{s} \equiv \frac{\mu_{B}}{e}\left(j_{\uparrow}-j_{\downarrow}\right)=V \frac{e \mu_{B}}{h} T \cos 2(\alpha-\beta) .
$$




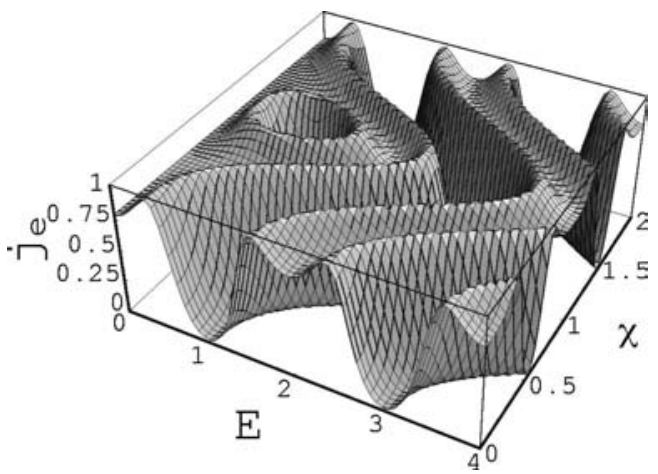

FIG. 2. Charge current $j_{e}$ with unit $V e^{2} / h$ variation with respect to electric field $E$ with unit $\hbar c / \mu a$ and its direction tilt angle $\chi$ with unit $\pi$.

From Eq. (9) it is evident that the transmission polarized spin current is controlled by tilt angle $\beta$ as well as by the modulation, analogous to the transmission charge current.

To see this modulation clearly, we introduce a dimensionless quantity $P_{z}$ to describe the polarization along the $S_{z}$ spin axis of current transmitted through the AC ring, which is defined by

$$
P_{z} \equiv \frac{j_{\uparrow}-j_{\downarrow}}{j_{\uparrow}+j_{\downarrow}}=\cos 2(\alpha-\beta) .
$$

$P_{z}$ is independent of mass $m$ and momentum $\hbar k$ of the incident charge carriers for large $k a$. This is an important advantage for device applications. $P_{z}$ is similar to the spin injection rate defined in ferromagnetic/semiconductor/ ferromagnetic heterostructures, ${ }^{23}$ and can be measured experimentally. $P_{z}$ is simply determined by $\alpha-\beta$ only. For incident state $\Psi_{i}$, the spin-polarized tilt angle is $2 \alpha$ and $P_{z}$ $=P_{0} \equiv \cos 2 \alpha$. We see that $P_{z}$ is modulated by $\beta$. In this case the current transmitted is modulated just by the AC phase and is spin independent. We plot $P_{z}$ vs $E$ and $\chi$ in Fig. 3 for the case of $\alpha=0$. From the definition of $\beta$, it is equal to $\chi$ when electric field $E$ is very large. It is obvious that $P_{z}$ approaches a constant with an increase of electric field strength for a certain angle. This mechanism is different from that in Datta and Das's proposal, where the difference in phase of two spin states determines spin precession. In this letter, the role of phase differences $\left(k_{1}^{ \pm}-k_{1}^{ \pm}\right) \pi a=\Phi_{\mathrm{AC}}^{ \pm}$of two spin eigenstates between the source and drain just controls the transmission coefficients and does not affect the spin polarizability.

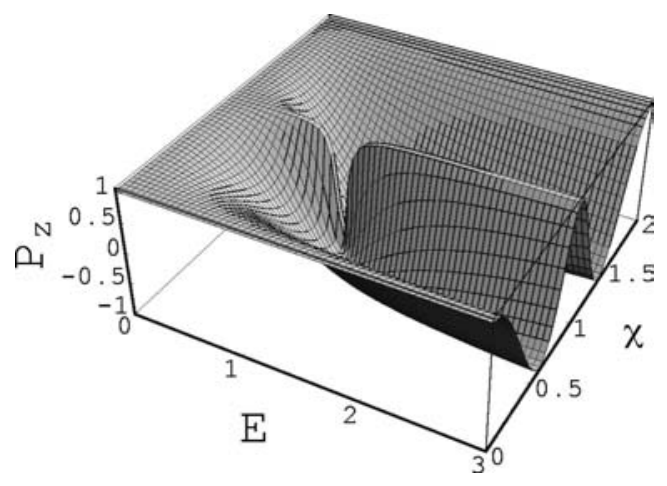

FIG. 3. Spin polarization of charge current along the $S_{z}$ spin axis $P_{z}$ with respect to electric field $E$ with unit $\hbar c / \mu a$ and its direction tilt angle $\chi$ with unit $\pi$.
This theory can be applied to a simple device to control the polarized spin current following Datta and Das' proposal. The semiconductor heterostructure is replaced by a conducting ring to connect the source and drain. Gate voltage is applied to control the electric field's effect on the ring, shown in Fig. 1(b). In this case one has $\mathbf{E}=-E \hat{z}$, i.e., $\chi=\pi / 2$. Assume a spin-up electron $(\alpha=0)$ is injected from the ferromagnetic source and passes through the $\mathrm{AC}$ ring. The tilt angle is simply written as $\beta=\arctan (\mu E a / \hbar c)$. The polarization orientation of the transmission current can be tuned by the electric field explicitly, i.e., $P_{z}=[1$ $\left.-(\mu E a / \hbar c)^{2}\right] /\left[1+(\mu E a / \hbar c)^{2}\right]$. For a large electric field of $E \gg \hbar c / \mu a$, we have $\beta \rightarrow \pi / 2$ and $P=-1$. The polarization orientation of the incident electron with spin up can be flipped into spin down. One of the advantages of the present device is that either the transmission probability or the modulation of polarization of current transmitted can be tuned by the electric field. Nitta et al. ${ }^{18}$ proposed a similar device in which the spin interference effect can be expected in the $\mathrm{AB}$ ring with uniform spin-orbit interaction. The phase differences between spin-up or -down waves in the ring are calculated, and the calculation shows that the $\mathrm{AC}$ dynamic phase is important in interference between opposite spin waves.

This work was supported by the Research Grant Council of Hong Kong under Grant No. HKU7088/01P, a CRCG grant from The University of Hong Kong and by the NNSFC-China, NSF of Guangdong Province, and ARC of ZSU. One of the authors (S.Q.S.) acknowledge Max-PlanckInstitute for Physics of Complex Systems, Dresden, Germany, for its support and hospitality, where part of the work was done.

${ }^{1}$ G. A. Prinz, Science 282, 1660 (1998); Phys. Today 48, 58 (1995).

${ }^{2}$ J. M. Kikkawa, Phys. Today 52, 33 (1999).

${ }^{3}$ S. A. Wolf, D. D. Awschalom, R. A. Buhrmann, J. M. Daughton, S. von Molnar, M. L. Roukes, A. Y. Chtchelkanova, and D. M. Treger, Science 294, 1488 (2001).

${ }^{4}$ S. Datta and B. Das, Appl. Phys. Lett. 56, 665 (1990).

${ }^{5}$ J. Nitta, T. Akazaki, H. Takayanagi, and T. Enoki, Phys. Rev. Lett. 78, 1335 (1997).

${ }^{6}$ D. Grundler, Phys. Rev. Lett. 84, 6074 (2000).

${ }^{7}$ J. C. Egues, G. Burkard, and D. Loss, Appl. Phys. Lett. 82, 2658 (2003); Phys. Rev. Lett. 89, 176401 (2002).

${ }^{8}$ J. Schliemann, J. C. Egues, and D. Loss, Phys. Rev. Lett. 90, 146801 (2003).

${ }^{9}$ X. F. Wang, P. Vasilopoulos, and F. M. Peeters, Appl. Phys. Lett. 80, 1400 (2002); Phys. Rev. B 65, 165217 (2002).

${ }^{10}$ A. A. Kiseley and K. W. Kim, Appl. Phys. Lett. 78, 775 (2001).

${ }^{11}$ A. G. Mal'shukov, V. V. Shlyapin, and K. A. Chao, Phys. Rev. B 66, 081311 (2002)

${ }^{12}$ D. Frustaglia, M. Henschel, and K. Richter, Phys. Rev. Lett. 87, 256602 (2001)

${ }^{13}$ Y. Aharonov and A. Casher, Phys. Rev. Lett. 53, 319 (1984).

${ }^{14}$ H. Mathur and A. D. Stone, Phys. Rev. Lett. 68, 2964 (1992).

${ }^{15}$ A. G. Aronov and Y. B. Lyanda-Geller, Phys. Rev. Lett. 70, 343 (1993).

${ }^{16}$ Y. S. Yi, T. Z. Qian, and Z. B. Su, Phys. Rev. B 55, 10631 (1997).

${ }^{17}$ T. Choi, S. Y. Cho, C. M. Ryu, and C. K. Kim, Phys. Rev. B 56, 4825 (1997).

${ }^{18}$ J. Nitta, F. E. Meijer, and H. Takayanagi, Appl. Phys. Lett. 75, 695 (1999).

${ }^{19}$ Y. Aharonov and J. Anandan, Phys. Rev. Lett. 58, 1593 (1987).

${ }^{20}$ R. Landauer, IBM J. Res. Dev. 1, 233 (1957); Philos. Mag. 21, 863 (1970).

${ }^{21}$ J. B. Xia, Phys. Rev. B 45, 3593 (1992).

${ }^{22}$ S. Griffith, Trans. Faraday Soc. 49, 345 (1953); 49, 650 (1953).

${ }^{23}$ M. Johnson, Phys. Rev. B 58, 9635 (1998); M. Johnson and R. H. Silsbee, ibid. 37, 5326 (1988). 